\title{
Generalized Entropy and Projection Clustering of Categorical Data
}

\author{
Dan A. Simovici, Dana Cristofor, and Laurentiu Cristofor \\ University of Massachusetts at Boston \\ Department of Mathematics and Computer Science, \\ Boston, Massachusetts 02446, USA, \\ $\{$ dsim, dana, laur\}@cs.umb.edu
}

\begin{abstract}
We generalize the notion of entropy for a set of attributes of a table and we study its applications to clustering of categorical data This new concept allows greater flexibility in identifying sets of attributes and, in a certain case, is naturally related to the average distance between the records that are the object of clustering. An algorithm that identifies clusterable sets of attributes (using several types of entropy) is also presented as well as experimental results obtained with this algorithm.
\end{abstract}

\section{Introduction}

In this paper we investigate clustering on categorical data, a problem that poses challenges that are quite distinct from numerical data clustering (see AGGR98 and [CFZ99]). This type of data can be encoded numerically; however, this introduces artificial total orderings on the domains of the attributes involved which have the potential to create artificial agglomerations that are meaningless from a practical point of view. Our approach to clustering is based on identifying projections that have sufficient concentrations of values (determined by certain types of entropies associated to attribute sets). The sets of records that are formed by grouping on these sets of attributes qualify as clusters if they do not differ too much on the remaining attributes; this requirement can be enforced by limiting the value of a certain generalization of conditional entropy. Our work extends the information-theoretical approach to clustering found in CFZ99.

Let $\mathbb{R}$ be the set of reals. The $k$-dimensional simplex is the set SIMPLEX $_{k-1}=$ $\left\{\left(p_{1}, \ldots, p_{k}\right) \in \mathbb{R}^{k} \mid p_{i} \geq 0\right.$ and $\left.p_{1}+\cdots+p_{k}=1\right\}$. A function $f: \mathbb{R} \longrightarrow \mathbb{R}$ is concave on a set $S \subseteq \mathbb{R}$ if $f(\alpha x+(1-\alpha) y) \geq \alpha f(x)+(1-\alpha) f(y)$ for $\alpha \in[0,1]$ and $x, y \in S$. The function $f$ is sub-additive (supra-additive) on $S$ if $f(x+y) \leq$ $f(x)+f(y)(f(x+y) \geq f(x)+f(y))$ for $x, y \in S$. In [CHH99] a concave impurity measure is defined as a concave real-valued function $i:$ SIMPLEX $_{k-1} \longrightarrow \mathbb{R}$ such that $i(\alpha \mathbf{p}+(1-\alpha) \mathbf{q})=\alpha i(\mathbf{p})+(1-\alpha) i(\mathbf{q})$ implies $\mathbf{p}=\mathbf{q}$ for $\alpha \in[0,1]$ and $\mathbf{p}, \mathbf{q} \in \operatorname{SIMPLEX}_{k-1}$ and, if $\mathbf{p}=\left(p_{1}, \ldots, p_{k}\right)$, then $i(\mathbf{p})=0$ if $p_{i}=1$ for some $i$, $1 \leq i \leq k$.

The corresponding frequency-weighted impurity measure is the real-valued function $I: \mathbb{N}^{k} \longrightarrow \mathbb{R}$ given by $I\left(n_{1}, \ldots, n_{k}\right)=N i\left(n_{1} / N, \ldots, n_{k} / N\right)$, where

D.A. Zighed, J. Komorowski, and J. Żytkow (Eds.): PKDD 2000, LNAI 1910, pp. 619-625, 2000. (C) Springer-Verlag Berlin Heidelberg 2000 
$N=\sum_{i=1}^{k} n_{i}$. Both the Gini impurity measure and the Shannon entropy can be generated using a simple one-argument concave function $f:[0,1] \longrightarrow \mathbb{R}$ such that $f(0)=f(1)=0$. In this paper we additionally require two extra conditions which are satisfied by many functions.

Definition 1. A concave function $f:[0,1] \longrightarrow \mathbb{R}$ is a generator if $f(0)=$ $f(1)=0, f$ is subadditive and for $\left(a_{1}, \ldots, a_{n}\right) \in \operatorname{SIMPLEX}_{n-1}$ and $k \in[0,1]$ we have $f\left(k a_{1}\right)+\cdots+f\left(k a_{n}\right) \leq k\left(f\left(a_{1}\right)+\cdots+f\left(a_{n}\right)\right)+f(k)$.

The monogenic impurity measure induced by the generator $f$ is the impurity measure generated by concave impurity measure $i$ of the form $i\left(p_{1}, \ldots, p_{k}\right)=$ $f\left(p_{1}\right)+\cdots+f\left(p_{k}\right)$, where $\left(p_{1}, \ldots, p_{k}\right) \in \operatorname{SIMPLEX}_{k-1}$.

It is easy to verify that such functions as $f_{\text {gini }}(p)=p-p^{2}, f_{\mathrm{sq}}(p)=\sqrt{p}-p$ $f_{\text {shannon }}(p)=-p \log p$, or $f_{\text {peak }}$ (given by $f_{\text {peak }}(p)=p$ for $0 \leq p \leq 0.5$ and $f_{\text {peak }}(p)=1-p$ for $\left.0.5<p \leq 1\right)$ satisfy the conditions that define generators. Since $f$ is concave it satisfies Jensen's inequality $f\left(p_{1}\right)+\cdots+f\left(p_{k}\right) \leq k f\left(\frac{1}{k}\right)$ for every $\left(p_{1}, \ldots, p_{k}\right) \in \operatorname{SIMPLEX}_{k-1}$ and this implies that the largest value of the sum $f\left(p_{1}\right)+\cdots+f\left(p_{k}\right)$ is achieved if and only if $p_{1}=\cdots=p_{k}=\frac{1}{k}$. Therefore, for the monogenic impurity measure generated by the function $f$ we have $0 \leq i\left(p_{1}, \ldots, p_{k}\right) \leq k f\left(\frac{1}{k}\right)$ for $\left(p_{1}, \ldots, p_{k}\right) \in$ SIMPLEX $_{k-1}$.

For relational terminology and notations see, for example, [ST95].

\section{Impurity of Sets and Partitions}

We introduce the notion of impurity of a subset of a set $S$ relative to a partition. In turn, this notion is used to define the impurity of a partition relative to another partition.

Definition 2. Let $f$ be a generator, $S$ be a set and let $\operatorname{PART}(S)$ be the set of all partitions of $S$. The impurity of a subset $L$ of $S$ relative to a partition $\pi \in \operatorname{PART}(S)$ is the monogenic impurity measure induced by $f$ :

$$
\operatorname{IMP}_{\pi}^{f}(L)=|L|\left(f\left(\frac{\left|L \cap B_{1}\right|}{|L|}\right)+\cdots+f\left(\frac{\left|L \cap B_{n}\right|}{|L|}\right)\right),
$$

where $\pi=\left\{B_{1}, \ldots, B_{n}\right\}$. The specific impurity of $L$ relative to $\pi$ is

$$
\operatorname{imp}_{\pi}^{f}(L)=\frac{\operatorname{IMP}_{\pi}^{f}(L)}{|L|}=f\left(\frac{\left|L \cap B_{1}\right|}{|L|}\right)+\cdots+f\left(\frac{\left|L \cap B_{n}\right|}{|L|}\right) .
$$

Jensen's inequality implies that the largest value of the impurity of a set $L \subseteq S$ relative to a partition $\pi=\left\{B_{1}, \ldots, B_{n}\right\}$ of $S$ is $|L| \cdot n \cdot f\left(\frac{1}{n}\right)$.

Theorem 1. Let $K, L$ be two disjoint subsets of the set $S$ and let $\pi \in \operatorname{PART}(S)$. Then, we have $\operatorname{IMP}_{\pi}^{f}(K \cup L) \geq \operatorname{IMP}_{\pi}^{f}(K)+\operatorname{IMP}_{\pi}^{f}(L)$.

Corollary 1. If $K, L$ are subsets of $S$ such that $K \subseteq L$ and $\pi \in \operatorname{PART}(S)$, then $\operatorname{IMP}_{\pi}^{f}(K) \leq \operatorname{IMP}_{\pi}^{f}(L)$.

Theorem 2. Let $\pi=\left\{B_{1}, \ldots, B_{n}\right\}$ and $\sigma=\left\{C_{1}, \ldots, C_{m}\right\}$ be two partitions of a set $S$. If $\pi \leq \sigma$, then $\operatorname{IMP}_{\sigma}^{f}(K) \leq \operatorname{IMP}_{\pi}^{f}(K)$ for every subset $K$ of $S$. 


\section{$3 \quad f$-Entropy}

Let $\tau=(T, H, \rho)$ be a table. For $X \subseteq H$ define the equivalence $\equiv_{X}$ by $u \equiv_{X} v$ if $u[X]=v[X]$; denote the corresponding partition of the set $\rho$ of tuples by $\pi_{X}$. Every block of $\pi_{X}$ corresponds to a distinct value of $\operatorname{aDom}_{\tau}(X)$. Therefore, $\left|\pi_{X}\right|=\left|\operatorname{aDom}_{\tau}(X)\right|$. If $U, V$ are two subsets of $H$ such that $U \subseteq V$, then $\pi_{V} \leq$ $\pi_{U}$.

Definition 3. Let $\tau=(T, H, \rho)$ be a table and let $X, Y$ be sets of attributes, $X, Y \subseteq H$. The $f$-entropy of $X$ is $\mathcal{H}^{f}(X)=\operatorname{imp}_{\pi_{X}}^{f}(\rho)$.

The conditional $f$-entropy of $X$ on $Y$ is $\mathcal{H}^{f}(X \mid Y)=\sum_{j=1}^{m} \frac{\left|C_{j}\right|}{|\rho|} \operatorname{imp}_{\pi_{X}}^{f}\left(C_{j}\right)$, where $\pi_{Y}=\left\{C_{1}, \ldots, C_{m}\right\}$.

In other words, the conditional entropy $\mathcal{H}^{f}(X \mid Y)$ is the average value of the specific impurity of the blocks of the partition $\pi_{Y}$ relative to the partition $\pi_{X}$.

Since $\pi_{\emptyset}=\{\rho\}$, it follows that $\mathcal{H}^{f}(X)=\mathcal{H}^{f}(X \mid \emptyset)$ for every set of attributes $X$. Therefore, we have $\mathcal{H}^{f}(X)=\sum_{i=1}^{n} f\left(\frac{\left|B_{i}\right|}{|\rho|}\right)$, where $\pi_{X}=\left\{B_{1}, \ldots, B_{n}\right\}$. For the conditional entropy we have $\mathcal{H}^{f}(X \mid Y)=\frac{1}{|\rho|} \sum_{j=1}^{m}\left|C_{j}\right| \sum_{i=1}^{n} f\left(\frac{\left|B_{i} \cap C_{j}\right|}{\left|C_{j}\right|}\right)$. The concavity of the generator $f$ implies that the largest value of the $f$-entropy of a set of attributes $X$ occurs when the blocks of the partition $\pi_{X}$ are of equal size. Therefore, we have $\mathcal{H}^{f}(X) \leq n f\left(\frac{1}{n}\right)$, where $n$ is the number of blocks of $\pi_{X}$, that is, the cardinality of the active domain of $X$.

Theorem 3. Let $X, X^{\prime}, Y, Y^{\prime}$ be sets of attributes of the table $\tau=(T, H, \rho)$. If $X \subseteq X^{\prime}$ and $Y \subseteq Y^{\prime}$, then $\mathcal{H}^{f}(X \mid Y) \leq \mathcal{H}^{f}\left(X^{\prime} \mid Y\right), \mathcal{H}^{f}(X \mid Y) \geq \mathcal{H}^{f}\left(X \mid Y^{\prime}\right)$ and $\mathcal{H}^{f}(X) \leq \mathcal{H}^{f}\left(X^{\prime}\right)$ for every generator $f$.

Theorem 4. Let $X, Y$ be two sets of attributes of a table $\tau=(T, H, \rho)$. We have $\mathcal{H}^{f}(X \mid Y) \leq \mathcal{H}^{f}(X)$ and $\mathcal{H}^{f}(X Y) \leq \mathcal{H}^{f}(X \mid Y)+\mathcal{H}^{f}(Y)$.

Corollary 2. For every sets of attributes $X, Y$ we have $\mathcal{H}^{f}(X Y) \leq \mathcal{H}^{f}(X)+$ $\mathcal{H}^{f}(Y)$.

\section{Clusters for Categorical Data}

Let $\tau=(T, H, \rho)$ be a table, $X$ be a set of attributes $X \subseteq H$ and assume that $\operatorname{aDom}_{\tau}(X)$ consists of $N$ elements, $\operatorname{aDom}_{\tau}(X)=\left\{x_{1}, \ldots, x_{N}\right\}$. Each block $B_{i}$ of $\pi_{X}$ corresponds to a value $x_{i}$ of the active domain of $X$ for $1 \leq i \leq N$, and $x_{i}$ appears under $X$ with the frequency $\frac{\left|B_{i}\right|}{|\rho|}$ for $1 \leq i \leq N$.

Let $d_{\tau, X}$ be a distance defined on $\operatorname{aDom}_{\tau}(X)$. The average distance between the elements of $\operatorname{aDom}_{\tau}(X)$ is $E\left(d_{\tau}(X)\right)=\frac{\sum_{i=1}^{N} \sum_{j=1}^{N}\left\{d_{\tau, X}\left(x_{i}, x_{j}\right)\left|B_{i}\right| \cdot\left|B_{j}\right|\right\}}{|\rho|^{2}}$. The next result connects one of the generalizations of entropy with the average distance and serves as a foundation for the applications of information-theoretical methods to clustering. 
Let $d_{\tau, X}$ be the distance on $\operatorname{aDom}_{\tau}(X)$ defined by: $d_{\tau, X}^{0,1}\left(x, x^{\prime}\right)=0$ if $x=x^{\prime}$; otherwise, $d_{\tau, X}^{0,1}\left(x, x^{\prime}\right)=1$ for $x, x^{\prime} \in \operatorname{aDom}_{\tau}(X)$.

Theorem 5. The average distance $E\left(d_{\tau, X}^{0,1}\right)$ equals $\mathcal{H}^{f_{\text {gini }}}(X)$.

Theorem 5 suggests that by limiting the entropy of a set of attributes $X$ we limit the average distance between the projections of the tuples of the table on $X$ and, therefore, increase our chances to find large clusters of tuples based on their similarity on $X$. A projection of a table $\tau$ on a set of attributes $X$ presents an interest for us if it satisfies two criteria:

1. The $f$-entropy $\mathcal{H}^{f}(X)$, referred to as the internal entropy of $X$ is limited. This insures that the values $X$-projections congregate towards a small number of clusters.

2. The impurity of each of the blocks of the partition $\pi_{X}$ relative to the partition $\pi_{H-X}$ is small. This insures that the tuples created by clustering the $X$ projection do not diverge excessively on the remaining attributes of the table. The definition of conditional $f$-entropy shows that such sets of attributes can be identified by limiting the value of the conditional entropy $\mathcal{H}^{f}(H-X \mid X)$, referred to as the external entropy of $X$ is limited.

Definition 4. Let $\tau=(T, H, \rho)$ be a table. An $(f, \alpha)$-clusterable set of attributes in $\tau$ is a set $X \subseteq H$ such that $\mathcal{H}^{f}(X) \leq \alpha$ and $X$ is maximal with this property, that is, $X \subset Y$ implies $\mathcal{H}^{f}(Y)>\alpha$.

The clusters determined by the $(f, \alpha)$-clusterable set $X$ are the groups of records that have equal values on $X$.

Next, we present an algorithm that can be used to identify clusterable sets of attributes. The algorithm is based on the monotonicity of the $f$-entropy shown in Corollary 1 Namely, if $U$ is a set of attributes such that $\mathcal{H}^{f}(U)>\alpha$, then for every set $V$ such that $U \subset V$ we have $\mathcal{H}^{f}(V)>\alpha$. Thus, once a set $U$ is disqualified from being $(f, \alpha)$-clusterable, no superset of $U$ can be $(f, \alpha)$-clusterable. Of course, if $U, U^{\prime}$ are $(f, \alpha)$-clusterable, the set $U U^{\prime}$ is a candidate for the collection KAttrSets and we apply the apriori-gen technique from $\left[\mathrm{AMS}^{+} 96\right]$ for idenfifying those sets that can be included in KAttrSets. If $X \subseteq X^{\prime}$, then, by Theorem 3 , we have $\mathcal{H}^{f}(H-X \mid X) \geq \mathcal{H}\left(H-X^{\prime} \mid X^{\prime}\right)$. Thus, to limit the external entropy, we focus on the maximal $(f, \alpha)$-clusterable sets of attributes.

We use three collections of sets of attributes: Candidates, KAttrSets and MaxAttrSets. At the completion of the algorithm shown in Figure 1 the collection MaxAttrSets will contain the $(f, \alpha)$-clusterable sets. The inputs of the algorithm are the generator function $f$ and the value $\alpha$ representing the limit of internal entropy. There are $\mathrm{N}$ attributes in our dataset. The external entropies of the sets of MaxAttrSets are also computed and we sort the maximal $(f, \alpha)$ clusterable sets on their external $f$-entropies.

The algorithm was run on a database extracted from the "agaricus-lepiota" dataset from UCI (http://www.ics.uci.edu/AI/ML/Machine-Learning.html) by 


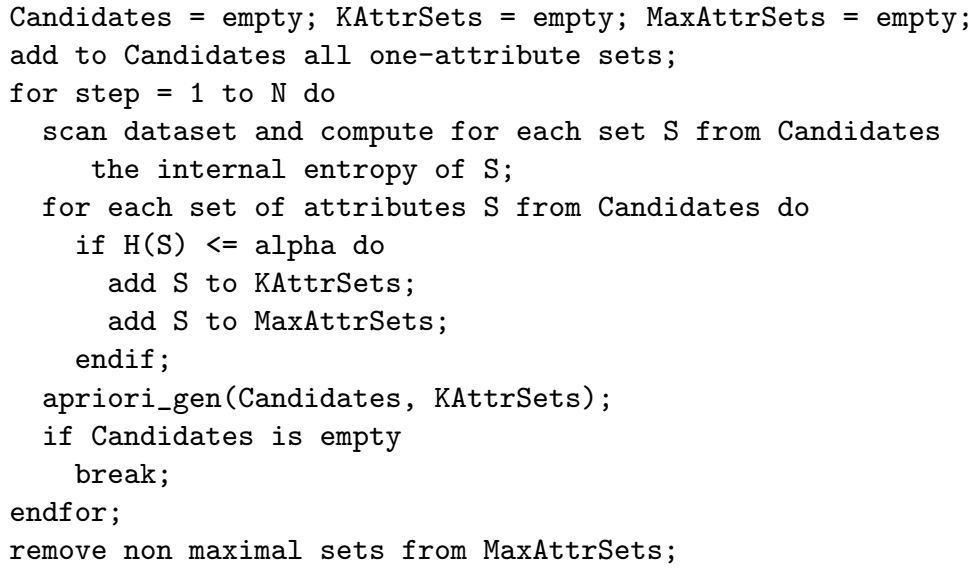

Fig. 1. Algorithm for Finding the $(f, \alpha)$-clusterable sets

retaining the first 15 attributes of a table documenting 23 attributes for a number of 8124 types of mushrooms. We experimented using three different function generators for different values of $\alpha: f_{\text {shannon }}, f_{\text {gini }}$, and $f_{\text {sq. }}$. The results are summarized below.

\begin{tabular}{|c|c|c|c|c|c|c|c|c|c|c|}
\hline \multirow[t]{2}{*}{$\alpha$} & \multicolumn{2}{|c|}{$f_{\text {gini }}$} & \multirow[t]{2}{*}{$\alpha$} & \multicolumn{2}{|c|}{$f_{\text {gini }}$} & \multirow[t]{2}{*}{$\alpha$} & \multicolumn{2}{|c|}{$f_{\text {shannon }}$} & \multicolumn{2}{|c|}{$f_{\mathrm{sq}}$} \\
\hline & time & sets & & time & sets & & time & sets & time & sets \\
\hline 0.5 & 1 & 5 & 0.92 & 59 & 92 & 1 & 1 & 5 & 3 & 14 \\
\hline 0.6 & 1 & 8 & 0.94 & 100 & 140 & 2 & 4 & 19 & 13 & 58 \\
\hline 0.7 & 3 & 16 & 0.96 & 231 & 156 & 4 & 56 & 140 & 97 & 233 \\
\hline 0.8 & 8 & 33 & 0.98 & 573 & 165 & 6 & 519 & 225 & 265 & 311 \\
\hline 0.9 & 42 & 93 & & & & 8 & 1541 & 110 & 527 & 311 \\
\hline
\end{tabular}

Elementary computations show that if $0 \leq p \leq 0.025$, then $f_{\text {gini }}(p) \leq$ $f_{\text {shannon }}(p) \leq f_{\mathrm{sq}}(p)$; for $0.025<p \leq 0.39$ we have $f_{\text {gini }}(p) \leq f_{\mathrm{sq}}(p) \leq f_{\text {shannon }}(p)$. Finally, if $0.39<p \leq 1$, we have $f_{\mathrm{sq}}(p) \leq f_{\text {gini }}(p) \leq f_{\text {shannon }}(p)$. Thus, $f_{\mathrm{sq}}$ "penalizes" the sets of attributes whose partitions contain small blocks (containing fewer than $2.5 \%$ of the set of all tuples); such sets are not good candidates for defining clusters of records. It is easy to see that if an attribute set partitions a set of tuples in $k$ blocks, then its maximum entropy would be $1-\frac{1}{k}$ for

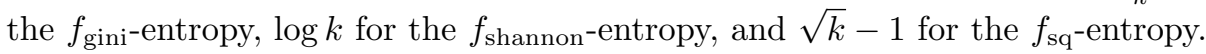
Considering this, by specifying a limit for the entropy of an attribute, we can get an approximative idea of how many large blocks its partition will have.

As an example of clusters determined by an $(f, \alpha)$-clusterable set of attributes, consider the attribute set bruises gill-attachment gill-spacing obtained when we used $f_{\text {shannon }}$ with $\alpha=2, f_{\text {gini }}$ with $\alpha=0.7$, and $f_{\text {sq }}$ with $\alpha=1$ and had a minimal external $f_{\mathrm{sq}}$-entropy. The clusters formed by grouping on these attributes contain 210, 3330, 1208, 3272 and 104 records, respectively, as shown in Figure 2 


\begin{tabular}{|c|c|c|c|c|c|}
\hline \multicolumn{3}{|c|}{ Set of attributes } & \multicolumn{3}{|c|}{ Block $B$} \\
\hline bruises & gill-attachment & gill-spacing & count & external clustering & $\operatorname{imp}_{\pi_{H-X}}^{f_{\text {sq }}(B)}$ \\
\hline no & attached & close & 210 & $\begin{array}{l}12 \times 16 \text { tuples } \\
18 \times 1 \text { tuple }\end{array}$ & 3.554 \\
\hline no & free & close & 3330 & $\begin{array}{l}72 \times 18 \text { tuples } \\
228 \times 6 \text { tuples } \\
48 \times 4 \text { tuples } \\
48 \times 2 \text { tuples } \\
18 \times 1 \text { tuple }\end{array}$ & 18.690 \\
\hline no & free & crowded & 1208 & $\begin{array}{l}216 \times 4 \text { tuples } \\
144 \times 2 \text { tuples } \\
56 \times 1 \text { tuple }\end{array}$ & 18.899 \\
\hline yes & free & close & 3272 & $\begin{array}{l}144 \times 12 \text { tuples } \\
120 \times 8 \text { tuples } \\
72 \times 4 \text { tuples } \\
148 \times 2 \text { tuples }\end{array}$ & 19.830 \\
\hline yes & free & crowded & 104 & $\begin{array}{l}48 \times 2 \text { tuples } \\
8 \times 1 \text { tuples }\end{array}$ & 6.440 \\
\hline
\end{tabular}

Fig. 2. Clusters Formed by Grouping

It is clear that the group with minimal impurity is obtained for bruises = 'no', gill-attachment = 'attached', and gill-spacing = 'closed'.
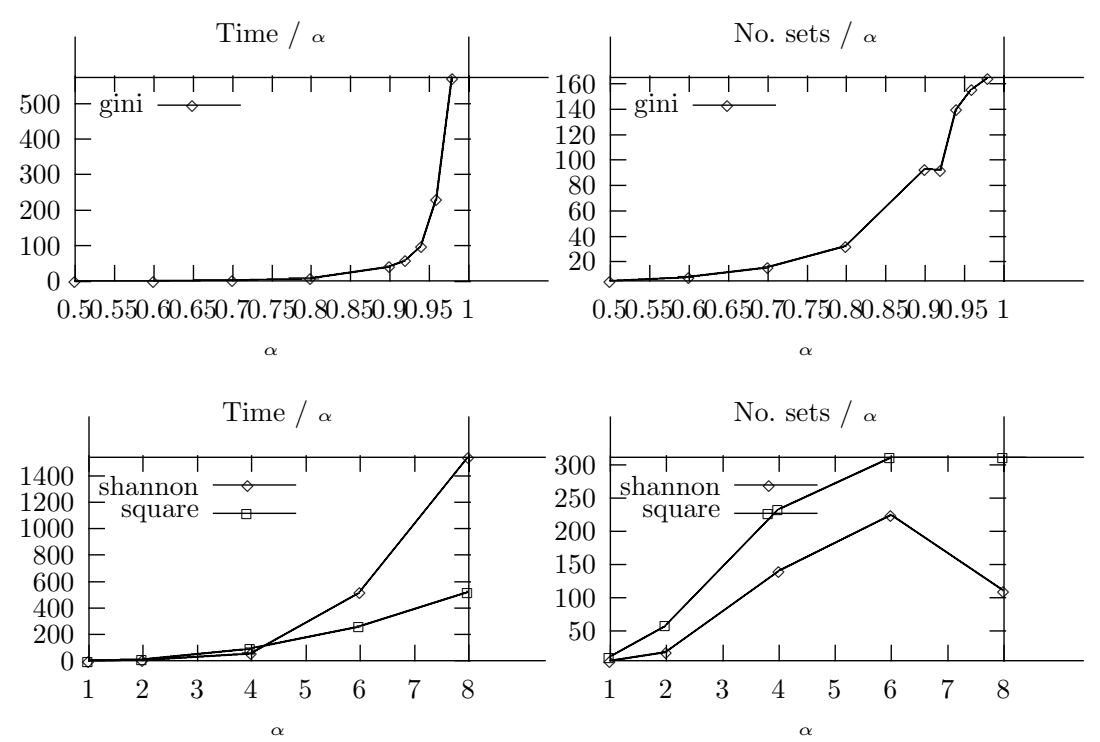


\section{Open Problems}

Our technique for identifying clusters could be improved by performing an initial step of eliminating all attributes that have a high $f$-entropy. These attributes can be considered as "noise" for the purpose of clustering, so by ignoring them we would clean the data and would make the values of external entropy more meaningful.

An investigation of classes of generator functions is necessary to determine the types of clusterable sets that are "favored" by these classes of generators.

The definition of clusters as groups of records determined by certain sets of attributes that have limited external impurities must be further refined and techniques that allow the user to choose a covering of the set of records by $(f, \alpha)$-clusters must be developed.

Also, an important open question is the axiomatization of the notion of $f$ entropy of an attribute set. Such an axiomatization should relax known axiomatizations of the notion of entropy (see [JS99]) to accomodate the generalizations discussed in this paper.

\section{References}

[AGGR98] R. Agrawal, J. Gehrke, D. Gunopulos, and P. Raghavan. Automatic subspace clustering for high dimensional data for data mining applications. In Proceedings of the 1998 ACM International Conference on Management of Data, pages 94-105, 1998.

$\left[\mathrm{AMS}^{+}\right.$96] R. Agrawal, H. Mannila, R. Srikant, H. Toivonen, and A. Inkeri Verkamo. Fast discovery of association rules. In U. M. Fayyad, G. Piatetsky-Shapiro, P. Smyth, and R. Uthurusamy, editors, Advances in Knowledge Discovery and Data Mining, pages 307-328. AAAI Press, Menlo Park, 1996.

[CFZ99] C. H. Cheng, A. W. Fu, and Y. Zhang. Entropy-based subspace clustering for mining numerical data. In Proceedings of the Fifth ACM SIGKDD International Conference on Knowledge Discovery and Data Mining, pages 84-93, 1999.

[CHH99] D. Coppersmith, S.J. Hong, and J.R.M. Hosking. Partitioning nominal attributes in decision trees. Data Mining and Knowledge Discovery, 3:197217,1999

[JS99] S. Jaroszewicz and D. A. Simovici. On axiomatization of conditional entropy. In Proceedings of the 29th International Symposium for MultipleValued Logic, pages 24-31, Freiburg, Germany, 1999.

[ST95] D. A. Simovici and R. L. Tenney. Relational Database Systems. Academic Press, New York, 1995. 